\title{
Calibration of the mirror system in the HERA-B RICH
}

\author{
Marko Starič ${ }^{a, *}$ and Peter Križan ${ }^{\text {b,a }}$ \\ a Jožef Stefan Institute, Ljubljana, Slovenia \\ ${ }^{\mathrm{b}}$ Faculty of Mathematics and Physics, University of Ljubljana, Slovenia
}

\begin{abstract}
The mirror system of the HERA-B RICH consists of two spherical and two planar mirrors, composed of altogether 116 mirror segments. Analysis of displacements of the Čerenkov ring center relative to the charged particle track, for given sphericalplanar segment pairs, leads to accurate information regarding the orientation of individual mirror segments. The method is described and the effect of applying the required corrections on the Cerenkov angle resolution of the HERA-B RICH is discussed.
\end{abstract}

Key words: Ring imaging Čerenkov counter, alignment, HERA-B

PACS: $29.40 . \mathrm{Ka}$

* Corresponding author

Email address: marko.staric@ijs.si (Marko Starič).

Preprint submitted to Elsevier 


\section{Introduction}

HERA-B (1) was a fixed target experiment (Fig. 1) at the HERA storage ring at DESY in Hamburg. The experiment used $920 \mathrm{GeV}$ protons from the beam halo and a set of eight thin ribbons, of different materials, as targets. The interaction rate was adjusted by moving the targets in or out of the beam halo (2). The experiment utilized a forward spectrometer capable of measuring interaction rates up to $40 \mathrm{MHz}$. The spectrometer consisted of a dipole magnet, a vertex detector (3) upstream and a main tracking system downstream of the magnet $(4 ; 5)$. Particle identification was performed by a Ring Imaging Čerenkov (RICH) detector (6) $)$, an electromagnetic calorimeter (7) and a muon detector system (8). In addition, the experiment included a sophisticated hardware trigger for lepton track pairs to record leptonic decays of $J / \psi$ particles. The large acceptance of the spectrometer coupled with high-granularity particle identification devices and a precision vertex detector allowed for detailed studies of multi-particle final states (9)-(16). By using targets of different materials, HERA-B was also able to study the dependence of various properties of proton-nucleus interactions as a function of atomic number.

The identification of pions, kaons and protons was performed by the RICH detector (6). The HERA-B RICH used atmospheric pressure $C_{4} F_{10}$ as Čerenkov radiator $(n=1.00137)$. The focusing of Čerenkov light was achieved with two spherical mirrors, tilted by $9^{0}$ in opposite directions (Fig. 1). Two planar mirrors then reflected the light to photon detectors at the top and bottom of the vessel containing the radiator gas. For the detection of Čerenkov photons multi anode PMTs (Hamamatsu R5900) were used. The inner part of the photon detector surface was equipped with 16-channel PMTs (16 x 4 mm x 4 mm) 
and the outer region with coarser granularity had 4-channel PMTs (4 x 8 mm x $8 \mathrm{~mm}$ ). To overcome the loss of photons due to inactive space between PMT photocathodes, a demagnifying lens system (17) was placed in front of each PMT.

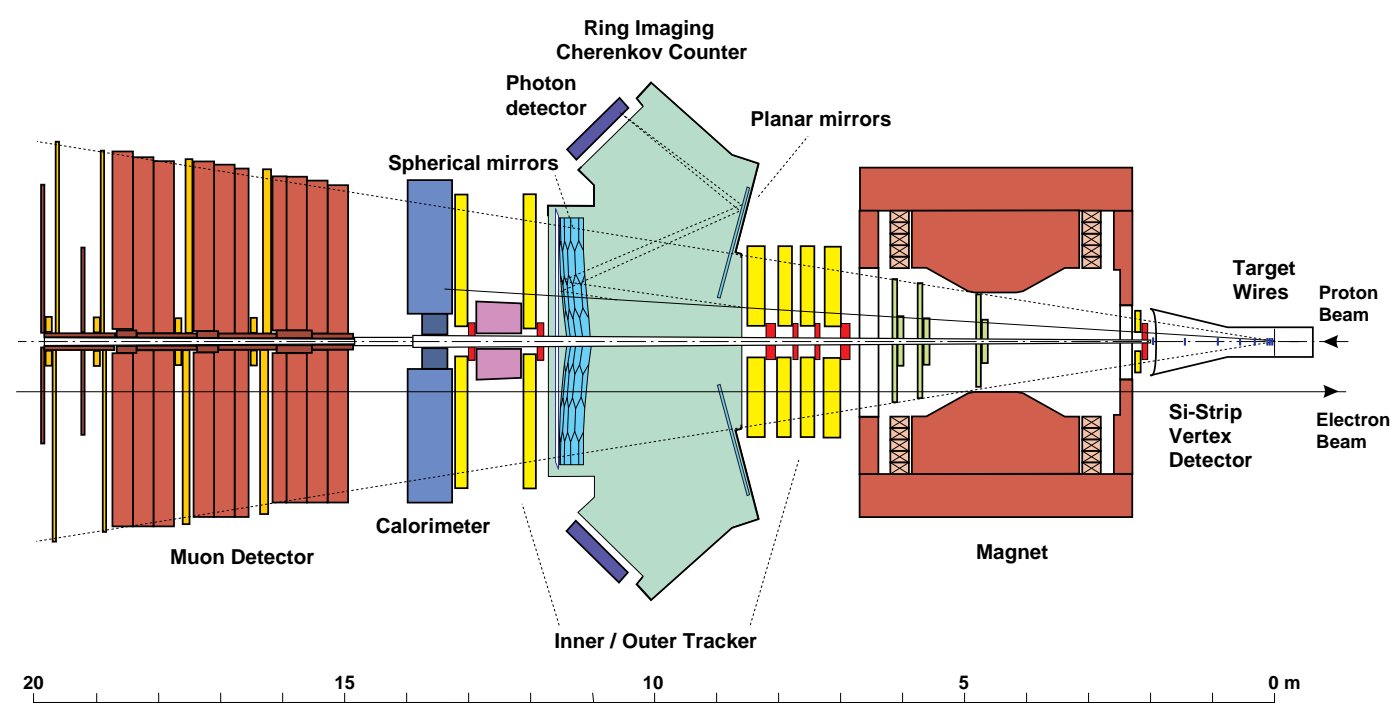

Fig. 1. A side view of the HERA-B detector. Photon paths in the RICH counter are indicated.

The particle identification capabilities of a RICH counter are determined by the resolution of the measured Cerenkov angle, which is given by the two main parameters of a RICH counter, the Čerenkov angle resolution due to a single photon and the number of detected photons per Čerenkov ring. The measured average number of detected photons for particles approaching the speed of light amounts to 33. It is in good agreement with the value expected from the data available on the quantum efficiency, mirror reflectivity, and transmissions of the vessel window and of the optical system (ㅁ) .

The main contributions to the single photon resolution (r.m.s.) come from the photon detector granularity $(0.50 \mathrm{mrad}$ and $0.93 \mathrm{mrad}$ for the regions covered by finer and coarser granularity PMTs respectively) and the dispersion in the 
radiator medium $(0.33 \mathrm{mrad})$. The optical error $(0.25 \mathrm{mrad})$ includes contributions from spherical aberration, mirror quality, and mirror alignment. The

contribution of multiple scattering in the RICH counter $\left(\frac{3.5 \mathrm{mrad}}{p(\mathrm{GeV} / \mathrm{c})}\right)$ becomes important at low momenta. The resulting expected single photon resolution, $0.65 \mathrm{mrad} \oplus \frac{3.5 \mathrm{mrad}}{\mathrm{p}(\mathrm{GeV} / \mathrm{c})}$ and $1.02 \mathrm{mrad} \oplus \frac{3.5 \mathrm{mrad}}{\mathrm{p}(\mathrm{GeV} / \mathrm{c})}$ for the regions covered by the two types of PMTs, does not include the contribution from the uncertainty in the track direction, which is given by other components of the HERA-B detector.

In order to reach and maintain the optimal performance of the RICH counter, elaborate alignment and calibration methods have to be used. For the optical system of the HERA-B RICH, a calibration method was used which is based on a procedure originally developed on simulated data (18).

\section{Calibration of the optical system}

The main imaging device of the HERA-B RICH is a spherical mirror placed inside the radiator vessel with the center of the sphere near the target and a radius of curvature of $11.4 \mathrm{~m}$. The mirror, a $6 \mathrm{~m}$ by $4 \mathrm{~m}$ rectangular cutout from the sphere, consists of 80 full or partial hexagons (see Fig. 2). To achieve a focal surface outside of the main particle flux ( $\pm 160 \mathrm{mrad}$ vertically), the mirror is split horizontally, and both halves are tilted by $9^{\circ}$ away from the beam-line. A set of two planar mirrors, composed of 18 rectangular elements each, translates the focal surface to the photon detector area above and below the radiator vessel (see Fig. 1). The 116 mirror segments are mounted on rigid, low mass support structures inside the radiator volume and can be individually adjusted by stepper motors from the outside. 


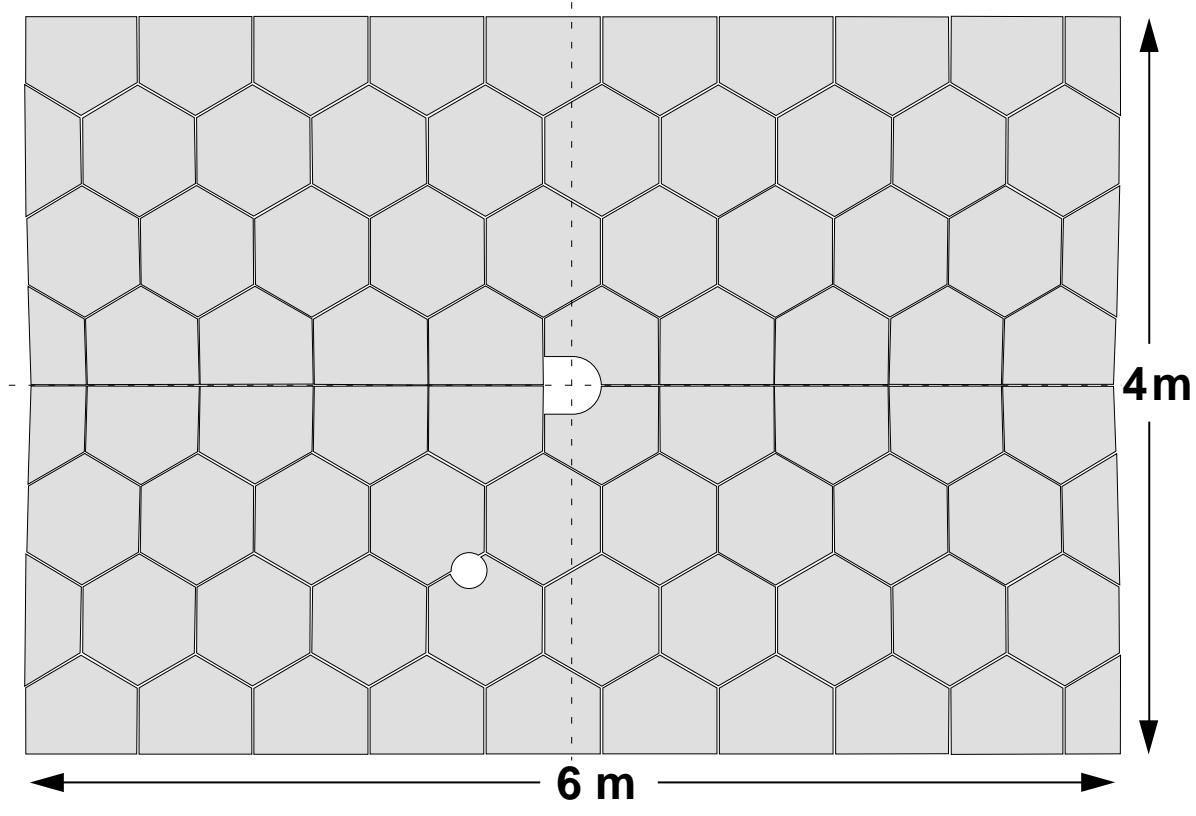

Fig. 2. Distribution of spherical mirror polygons. The holes in the array are for the proton and electron beam pipes.

All mirrors were first aligned after installation by surveying them inside the vessel. During the data taking periods, the mirror system was calibrated by making use of recorded events. By comparing the charged particle track direction, obtained from the Cerenkov rings due to a particular spherical-planar mirror pair, to the track direction obtained from other detectors of HERA-B, the calibration parameters of individual mirror segments, as well as those of the entire RICH counter, could be extracted. For this purpose, various data sets have been used. With the magnetic field turned off, the direction of the straight tracks was accurately given by the target wire position and the centroid of the cluster in the electromagnetic calorimeter. With the magnetic field turned on, the tracks were determined by the tracking system. In order to reduce the uncertainty in track direction due to multiple Coulomb scattering, only those tracks belonging to particles with energy above $5 \mathrm{GeV}$ were used. 


\subsection{The calibration method}

Assume that one or both mirrors in a particular spherical-planar mirror pair are not well aligned. In such a case, the measured Čerenkov ring, due to photons reflected on that pair, will be displaced relative to the direction of the charged particle, which is taken to be reflected on ideal mirror positions. For small displacements $a$, the azimuthal dependence of Čerenkov angle for photons on a given ring is parameterized as (Fig. 3):

$$
\theta_{c}=\theta_{0}+a \cos \left(\phi_{c}-\phi_{0}\right)=\theta_{0}+\Delta \Phi \cos \phi_{c}+\Delta \lambda \sin \phi_{c}
$$

where $\theta_{0}$ is the nominal value of the Čerenkov angle. The parameters $\Delta \Phi=$ $a \cos \phi_{0}$ and $\Delta \lambda=a \sin \phi_{0}$ roughly correspond to rotations of the mirrors around vertical and horizontal axes. A rotation of a spherical mirror segment by $\delta$ around the vertical and horizontal axes results in $\Delta \Phi=\delta$ and $\Delta \lambda=\delta$, respectively, while the same rotation of the planar mirror results in $\Delta \Phi \approx \delta / 2$ and $\Delta \lambda \approx \delta / 2$ (see Fig. 4 ).
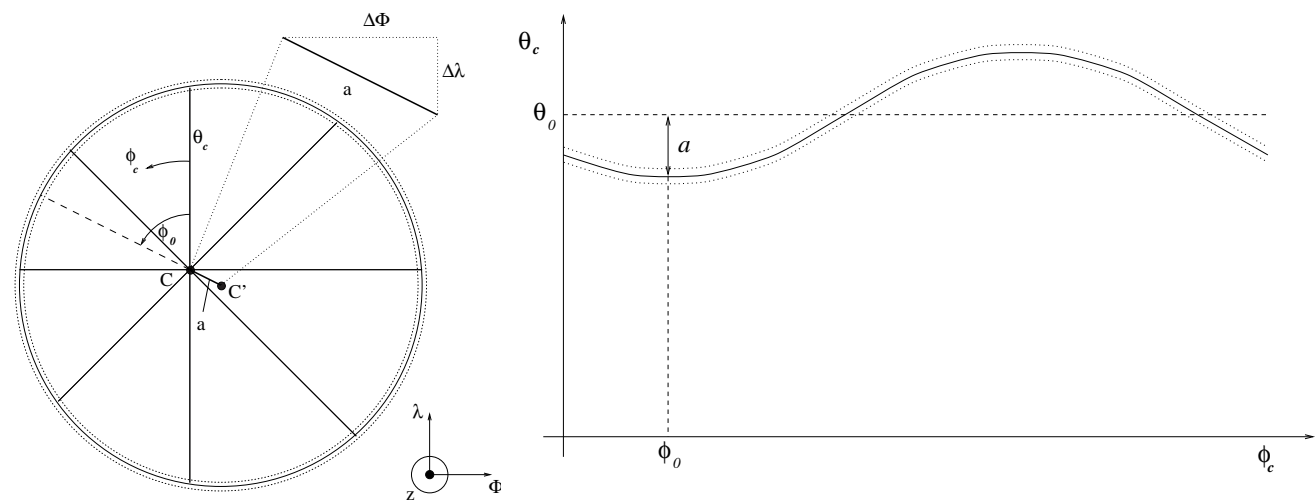

Fig. 3. For misaligned mirrors, the center of the measured ring $\mathrm{C}^{\prime}$ is displaced relative to the extrapolated charged particle track direction $\mathrm{C}$ (left). In such a case, the measured Čerenkov angle $\theta_{c}$ depends on the azimuth $\phi_{c}$ of the photon hit (right). 
The method obviously relies on accumulating a sufficient number of photons, which have been reflected on a particular spherical-planar mirror pair. For each track-photon pair, the photon has been traced from two points on the charged particle track to the photon hit position (Fig. 4). The first point is the particle entry into the radiator, the second is directly in front of the spherical mirror. If both rays at a given azimuthal angle are reflected from the same spherical-planar mirror pair, such a photon hit is taken as a valid data point on the Čerenkov ring, relevant for that mirror pair. In other words, a photon hit is valid for our analysis if the corresponding photon would have been reflected from the same spherical-planar mirror pair, regardless of the point on the charged particle trajectory from which it might have originated. In the following we refer to such hits as calibration hits.

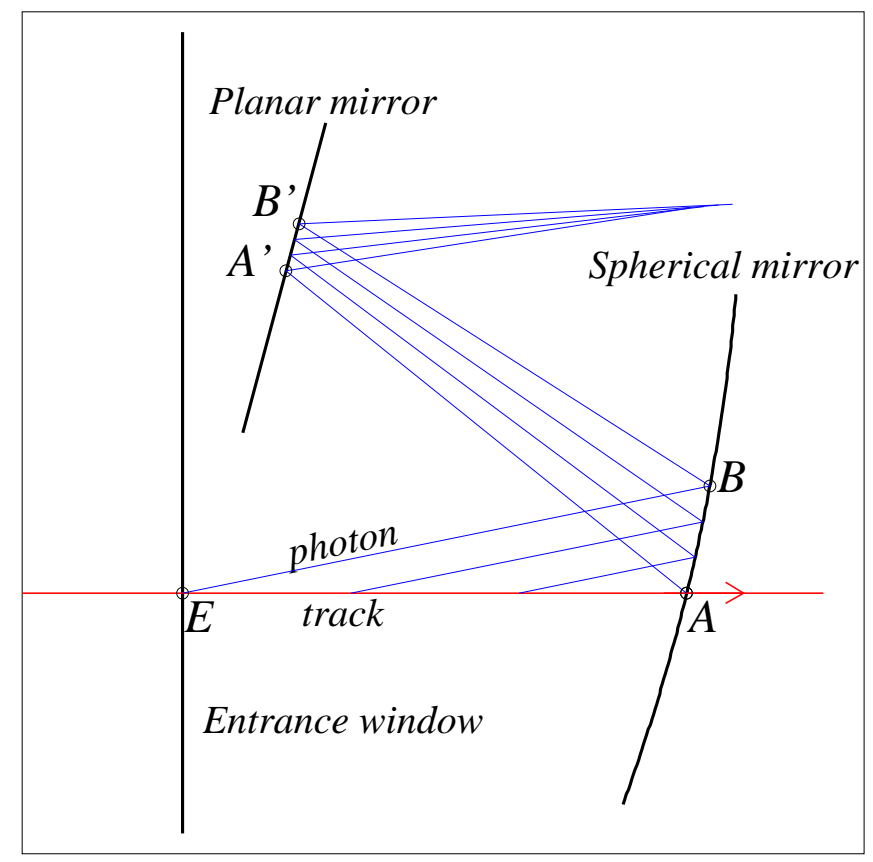

Fig. 4. For calibration of mirror orientations, only those photon hits have been used, for which the corresponding photons could have been reflected only from a given spherical-planar mirror pair, regardless of the point on the particle track, from which the photon was emitted. 
Figure 5 shows the distribution of such hits for two particular combinations of a spherical and two planar mirrors. The two-dimensional histograms on the left hand side of the figure represent the number of calibration hits as a function of Čerenkov angle difference $\Delta \theta_{c}=\theta_{c}-\theta_{c}^{\pi}(p)$ and azimuthal angle $\phi_{c}$. The difference to the nominal Čerenkov angle $\theta_{c}^{\pi}(p)$ of a pion at the measured momentum $p$ is chosen in order to suppress the momentum dependence; the pion hypothesis is chosen since the majority of tracks correspond to pions. An accumulation of hits at about $\Delta \theta_{c}=0$ can be seen, with some modulation dependent on azimuthal angle $\phi_{c}$. For each of 50 slices in $\phi_{c}$, the distribution was fitted with a Gaussian for the peak and a polynomial background (Fig. 6). The Čerenkov peak position as a function of azimuthal angle (right hand side of Fig. 5) is then fitted with the function $\Delta \Phi \cos \phi_{c}+\Delta \lambda \sin \phi_{c}+C$, and the rotation angles $\Delta \Phi$ and $\Delta \lambda$ are obtained for the given mirror pain 1 .

Close to mirror boundaries, parts of a ring could be shared by different combinations of spherical and planar mirrors, which permits relative calibration of adjacent mirrors.

\subsection{Extraction of calibration parameters of individual mirror segments}

The measured displacement $(\Delta \Phi, \Delta \lambda)$ for a pair of mirrors is equal to the sum of unknown contributions from the corresponding spherical and planar mirror segments $k$ and $j$ :

$$
\Delta \Phi_{k j}=\Delta \Phi_{k}^{s p h}+\Delta \Phi_{j}^{p l}
$$

$\overline{1}$ The parameter $C$ is a constant to account for a possible bias in the Čerenkov angle measurement. 

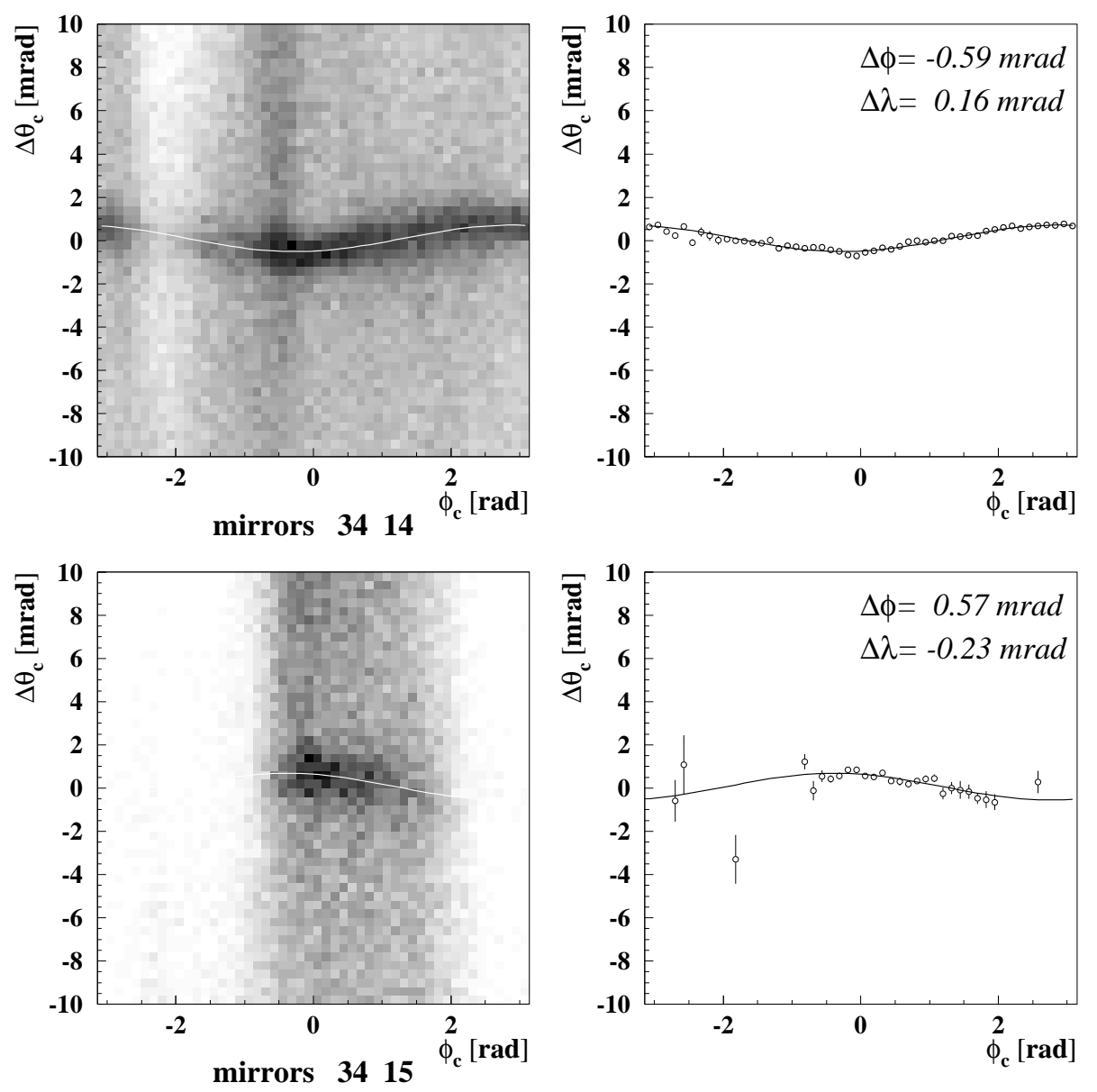

Fig. 5. Distribution of hits in the $\Delta \theta_{c}, \phi_{c}$ plane for two combinations of a spherical and two planar mirror segments. Raw data are shown on the left side, and the ring peak position from the fit in each $\phi_{c}$ slice is shown on the right.

$$
\Delta \lambda_{k j}=\Delta \lambda_{k}^{s p h}+\Delta \lambda_{j}^{p l}
$$

This represents a system of $2 m$ linear equations for the $2 n$ unknown parameters, where $m$ is the number of all measured segment combinations $(k, j)$, and $n$ is the number of mirror segments. As any given mirror segment contributes to more than one combination, there are more equations than there are unknowns. Arranging the measured $\Delta \Phi_{k j}$ and $\Delta \lambda_{k j}$ into vectors $b_{i}^{\Phi}$ and $b_{i}^{\lambda}$, with dimensions equal to $m$, the system of equations may be written for each of 


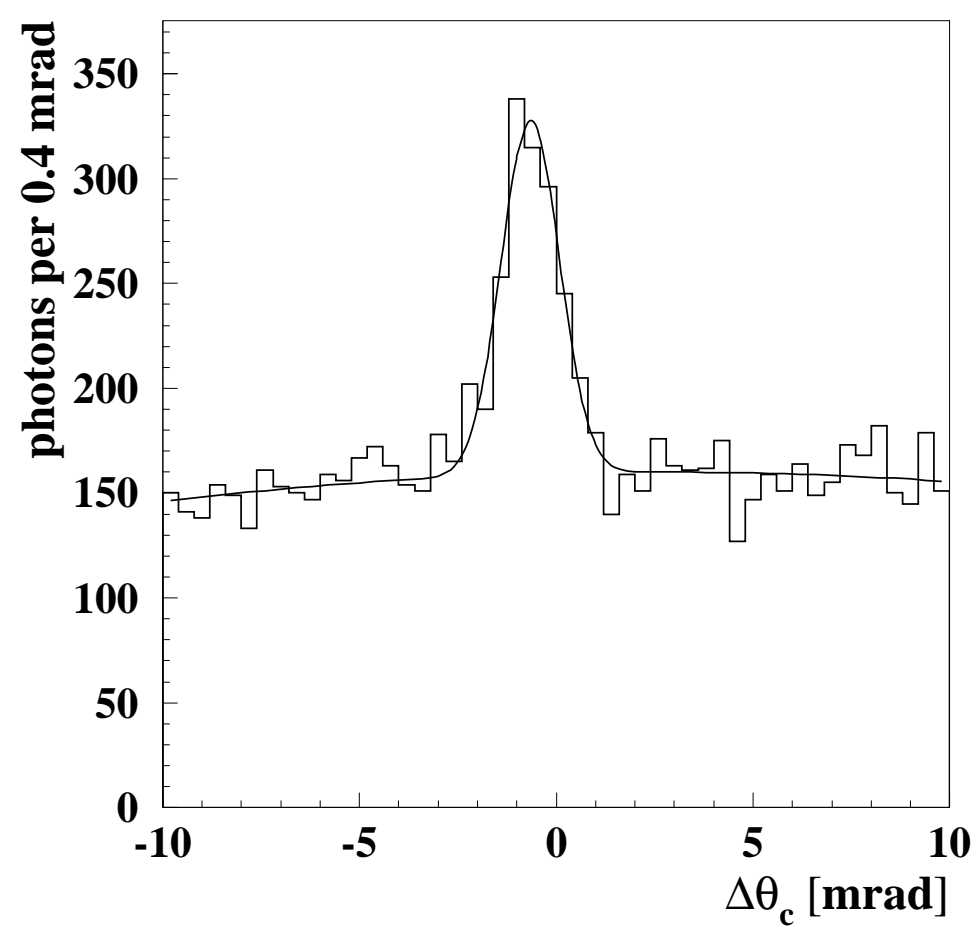

Fig. 6. Distribution of hits in $\Delta \theta_{c}$ for one of the $\phi_{c}$ slices. The result of the fit is superimposed.

the detector halves as

$$
b_{i}^{\Phi}=\sum_{l=1}^{n} A_{i l} u_{l}^{\Phi}, \quad b_{i}^{\lambda}=\sum_{l=1}^{n} A_{i l} u_{l}^{\lambda}, \quad i=1, \ldots, m,
$$

where $u_{l}^{\Phi}$ and $u_{l}^{\lambda}$ are the unknown contributions to the rotation $(\Delta \Phi, \Delta \lambda)$ of a particular mirror segment. Note that the matrix $A_{i l}$ is of a particular simple form; in a given row it only has 1 at two places (given by the indices of the spherical and planar segments, Eqs. 2 and 3), and is otherwise equal to zero.

Note that we have to add an additional condition to fully determine the system: if all spherical mirrors are turned by $\delta$, and all planar mirrors by $\approx-2 \delta$, one arrives at the same ring displacements (vectors $b_{i}^{\Phi}$ and $b_{i}^{\lambda}$ ). Rather than fixing the displacement of one mirror in the lower and one in the upper half to zero (one of the possibilities), we require of the solution to minimize the 
necessary mirror readjustments,

$$
\sum_{i_{s}} u_{i_{s}}^{\Phi}-\sum_{i_{p}} u_{i_{p}}^{\Phi}=0, \quad \sum_{i_{s}} u_{i_{s}}^{\lambda}-\sum_{i_{p}} u_{i_{p}}^{\lambda}=0
$$

The first sum in each of the equations runs over all spherical mirror segments and the second over all planar ones in a given detector half. We include these two equations in the system of equations 4 , and get two systems with $m+1$ equations,

$$
b_{i}^{\Phi}=\sum_{l=1}^{n} A_{i l}^{\prime} u_{l}^{\Phi}, \quad b_{i}^{\lambda}=\sum_{l=1}^{n} A_{i l}^{\prime} u_{l}^{\lambda}, \quad i=1, \ldots, m+1
$$

where $A^{\prime}$ replaces $A$ to account for the additional equation.

The systems of equations, Eq. 6, are solved by requiring that the properly weighted sum of squares of deviations of the left hand side from the right hand side for each of the two systems is minimal,

$$
\sum_{i=1}^{m+1} \frac{\left(\sum_{l=1}^{n} A_{i l}^{\prime} u_{l}-b_{i}\right)^{2}}{\sigma_{i}^{2}}=\min
$$

where the terms are weighted by the inverse square of the error $\sigma_{i}$ in the measurement 2 of $b_{i}$. Here $u_{l}$ and $b_{i}$ denote either $u_{l}^{\Phi}$ and $b_{i}^{\Phi}$ or $u_{l}^{\lambda}$ and $b_{i}^{\lambda}$. The resulting linear system,

$$
\sum_{l=1}^{n} \sum_{i=1}^{m+1} \frac{A_{i l}^{\prime} A_{i j}^{\prime}}{\sigma_{i}^{2}} u_{l}=\sum_{i=1}^{m+1} \frac{A_{i j}^{\prime}}{\sigma_{i}^{2}} b_{i}
$$

is readily solved,

$$
u_{k}=\sum_{j=1}^{n}\left(B^{-1}\right)_{k j} \sum_{i=1}^{m+1} \frac{A_{i j}^{\prime}}{\sigma_{i}^{2}} b_{i} .
$$

$\overline{2}$ For the two additional equations 5 , a value of $\sigma_{i}=0.01 \mathrm{mrad}$ was assumed; no influence was found when this value was varied. 
Here we have defined a new matrix

$$
B_{l j}=\sum_{i=1}^{m+1} \frac{A_{i l}^{\prime} A_{i j}^{\prime}}{\sigma_{i}^{2}}
$$

which is symmetric, so that its inverse $\mathbf{B}^{-\mathbf{1}}$ is easy to calculate. The resulting errors on $u_{k}$ are given by

$$
\sigma_{u_{k}}^{2}=\sum_{i=1}^{m+1}\left(\sum_{j=1}^{n}\left(B^{-1}\right)_{k j} \frac{A_{i j}^{\prime}}{\sigma_{i}}\right)^{2} .
$$

\subsection{Calibration results}

Having solved the system of equations for angular displacements of individual mirror segments, the quality of the new alignment is checked on the data, by examining the Čerenkov angle resolution before and after applying the new calibration parameters, $\Delta \Phi_{k}^{s p h}, \Delta \lambda_{k}^{s p h}, \Delta \Phi_{j}^{p l}$, and $\Delta \lambda_{j}^{p l}$. The result is shown in Fig. 7; the improvement in resolution is clearly visible.

To check the resulting alignment of the optical system, we have reanalyzed the data after having applied the calculated corrections. As expected, the new corrections were consistent with zero.

To study possible systematic effects, we have investigated different data sets, recorded under different conditions. We found good agreement of the results with two different gas radiators, when freon $\left(\theta_{c}=52 \mathrm{mrad}\right)$ was replaced with nitrogen $\left(\theta_{c}=25 \mathrm{mrad}\right)$. A similar analysis was also performed on the data recorded without magnetic field. Again the analysis yielded results that are consistent with the values deduced from the data with magnetic field. We also found that the alignment parameters did not change over extended periods of time, which testifies to stable mirror positions. 

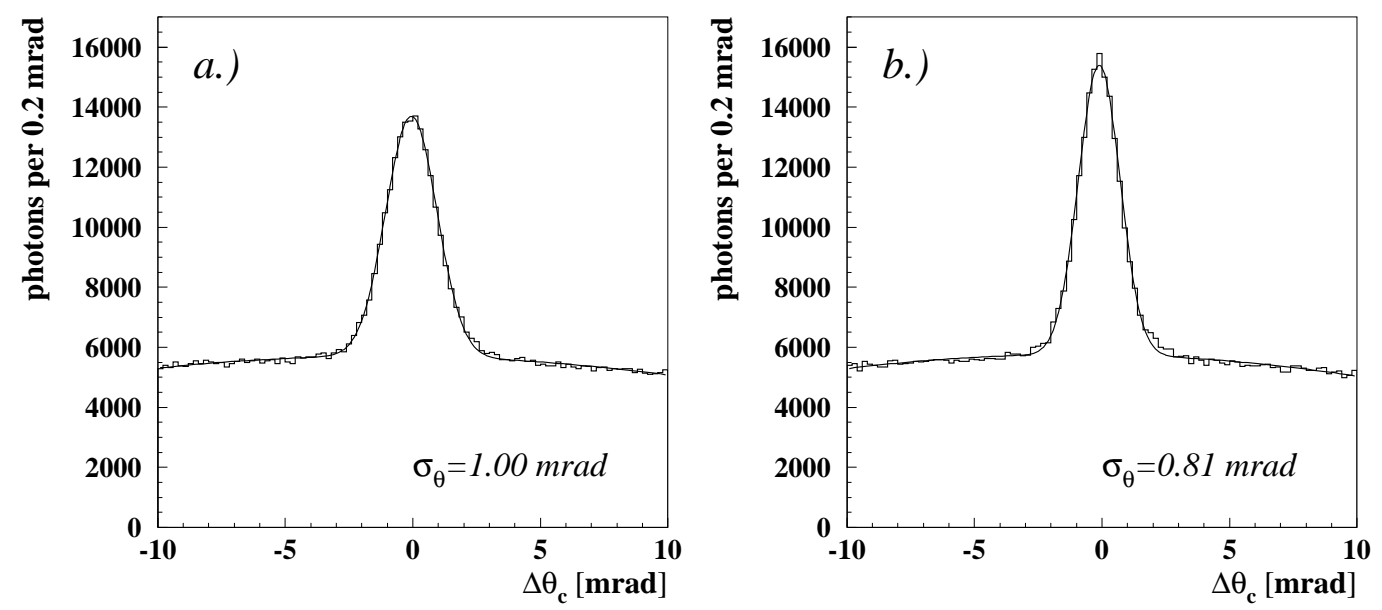

Fig. 7. The distribution of photon hits with respect to the corresponding Cerenkov angle difference $\Delta \theta_{c}$ before (a) and after (b) applying the correction obtained from the calibration of mirrors.

Possible systematic effects were also checked by using Monte Carlo generated events, where all mirror segments were assumed to be perfectly aligned. Although the resulting parameters are all consistent with zero, small systematic effects at the level of $0.1 \mathrm{mrad}$ could not be excluded due to limited statistics.

\section{Conclusions}

A method was developed for determination of the alignment of the RICH counter relative to other parts of the spectrometer, either the system of tracking chambers or the electromagnetic calorimeter. The method was tested on various sets of real data, recorded with and without magnetic field, as well as on simulated data. By applying alignment corrections for each mirror segment as derived by this method, a significant improvement in the resolution of the Čerenkov angle measurement could be obtained.

The HERA-B experiment finished data taking in spring 2003. During its five 
years of operation, the HERA-B RICH has proved reliability and stability of all its components and especially of the multi anode PMTs. The low noise, high rate capability and excellent long term stability of these devices enabled excellent operation in the hostile environment of a hadron machine. The HERA-B RICH identifies pions, kaons and protons essentially in the entire kinematic range of the HERA-B experiment with the identification efficiencies as large as 90\% and mis-identification probability at the $1 \%$ level $(6 ; 19)$. With kaon and proton identification the combinatorial background is in some cases reduced by more than 3 orders of magnitude. Several physics analyses would not have been possible without the excellent performance of the RICH particle identification system $(19 ; 9 ; 15 ; 16)$.

\section{References}

[1] T. Lohse et al., Proposal for HERA-B, DESY PRC-94/02, May 1994.

[2] K. Ehret, Nucl. Instrum. Meth. A 446 (2000) 190.

[3] C. Bauer et al., Nucl. Instrum. Meth. A 501 (2003) 39.

[4] W. Gradl, Nucl. Instrum. Meth. A 461 (2001) 80.

[5] H. Albrecht et al., Nucl. Instrum. Meth. A 555 (2005) 310; ibid. A 541 (2005) 610; ibid. A 576 (2007) 312.

[6] I. Arinyo et al., Nucl. Instrum. Meth. A 516 (2004) 445.

[7] G. Avoni et al., Nucl. Instrum. Meth. A 461 (2001) 332.

[8] V. Eiges et al., Nucl. Instrum. Meth. A 461 (2001) 104.

[9] I. Abt et al. (HERA-B Collaboration), Phys. Rev. Lett. 93 (2004) 212003.

[10] I. Abt et al. (HERA-B Collaboration), Phys. Lett. B 596 (2004) 173.

[11] I. Abt et al. (HERA-B Collaboration), Phys. Rev. D 73 (2006) 052005.

[12] I. Abt et al. (HERA-B Collaboration), Phys. Lett. B 638 (2006) 13. 
[13] I. Abt et al. (HERA-B Collaboration), Phys. Lett. 638 (2006) 407.

[14] I. Abt et al. (HERA-B Collaboration), Eur. Phys. J. C 49 (2007) 545.

[15] I. Abt et al. (HERA-B Collaboration), Eur. Phys. J. C 50 (2007) 315.

[16] I. Abt et al. (HERA-B Collaboration), hep-ex/0708.1443, accepted for publication in Eur. Phys. J. C.

[17] D. R. Broemmelsiek, Nucl. Instrum. Meth. A 433 (1999) 136.

[18] A. Gorišek, P. Križan, S. Korpar and M. Starič, Nucl. Instrum. Meth. A 433 (1999) 408.

[19] M. Starič, Nucl. Instrum. Meth. A 533 (2005) 210. 